\title{
Téoros
}

Revue de recherche en tourisme

\section{Thématiques de la recherche en tourisme menée par le CIFORT et ses membres}

\section{Boualem Kadri}

Volume 23, numéro 1, printemps 2004

URI : https://id.erudit.org/iderudit/1071374ar

DOI : https://doi.org/10.7202/1071374ar

Aller au sommaire du numéro

Éditeur(s)

Université du Québec à Montréal

ISSN

0712-8657 (imprimé)

1923-2705 (numérique)

Découvrir la revue

Citer cette note

Kadri, B. (2004). Thématiques de la recherche en tourisme menée par le

CIFORT et ses membres. Téoros, 23(1), 86-88. https://doi.org/10.7202/1071374ar d'utilisation que vous pouvez consulter en ligne.

https://apropos.erudit.org/fr/usagers/politique-dutilisation/ 
Centre international de formation et recherche en tourisme

\section{Thématiques de la recherche en tourisme menée par le CIFORT et ses membres}

\section{Boualem Kadri}

Denvergure acquise aujourd'hui par le tourisme suscite de plus - en plus d'intérêt de la part de nombreux acteurs institutionnels dans le monde : les grandes organisations internationales qui le considèrent comme un outil stratégique du développement; les institutions de formation supérieure du Nord qui participent au développement des pays les moins avancés, tout en posant une réflexion sur le phénomène touristique (tentative d'élaboration d'un champ disciplinaire distinct). Comment le Centre international de formation et de recherche en tourisme (CIFORT), outil d'internationalisation de l'UQAM en matière de tourisme, participe-t-il par le biais ses membres à cette reconnaissance scientifique du tourisme ?

Nous présentons dans ce document un aperçu de quelques thématiques développées ainsi que quelques projets de recherche menés par le CIFORT et ses membres pour les années 2004-2006.

\section{Les thématiques et les projets de recherche}

\section{La fracture numérique}

La fracture numérique est un phénomène mondial qui exprime des enjeux importants pour les sociétés développées, notamment en ce qui concerne l'écart dans le savoir (Castells, 2002). Il s'agit ici de la dimension de l'éducation et de la formation, où les technologies modifient les pédagogies d'enseignement et forcent à l'adaptation. Pour les pays industrialisés, l'enjeu mondial est celui du positionnement dans l'économie du savoir représentée par l'université. Pour les pays en développement, l'objectif est de réduire l'écart avec les pays développés en termes de connaissances, en utilisant le potentiel de l' $e$-formation.

Cette thématique globale a été étudiée par François Bédard et Boualem Kadri dès 2000. Les résultats de leurs travaux ont fait l'objet d'une publication parue aux Presses de l'Université du Québec, sous le titre Réduction de la fracture numérique en tourisme - Le rôle des grandes organisations internationales.

Deux nouveaux projets sur la même thématique ont été lancés récemment. Le premier projet porte sur la fracture numérique intrauniversitaire et le second sur le développement de la formation à distance dans les pays en développement.

La fracture numérique intra universitaire

Une première enquête scientifique sur les technologies de l'infor- mation et de la communication (TIC) a été réalisée auprès des enseignants de l'UQAM en 2002. Cette enquête, qui portait sur les attitudes et les perceptions vis-à-vis des TIC, a permis d'identifier une fracture numérique en ce qui concerne les personnels enseignants de l'UQAM (professeurs et chargés de cours) (Bédard et Géronimi, 2003). Pour approfondir cette problématique, un projet de recherche sur la fracture numérique intrauniversitaire a été déposé au Fonds québécois de la recherche sur la société et la culture (FQRSC). Ce projet s'inscrit dans le contexte du phénomène de la fracture numérique observée dans les institutions de formation supérieure. Ces institutions (universités, centres de recherche), qui constituent l'élément central de l'économie du savoir, sont confrontées à la mutation des formes d'acquisition et de transmission du savoir imposée par les technologies de l'information et de la communication. L'objectif principal est d'analyser comment les enseignants et l'institution se positionnent dans le processus de changement et d'intégration des technologies. Cela permettra de dégager une typologie des comportements et des orientations stratégiques des enseignants, devant aboutir à l'élaboration des stratégies d'intégration et d'adaptation.

\section{Développement d'un modèle de coopération internationale en e-formation}

En s'inspirant des objectifs de l'entente de coopération signée en 1999 entre l'Organisation mondiale du tourisme et le Groupe de la Banque mondiale, des chercheurs du CIFORT ont initié en 2000 un projet de recherche visant à développer un modèle de coopération en e-formation en tourisme entre les membres du Conseil d'éducation de l'OMT et des institutions de formation supérieure en tourisme venant de pays en développement membres de l'OMT. Le concept initial de coopération en e-formation a été présenté lors d'un colloque international parrainé par l'OMT tenu à l'UQAM en 2001, dans le cadre de la conférence ENTER de la International Federation of Information Technology and Travel and Tourism. À l'automne 2001, un rapport préliminaire de ce projet a été présenté à Séoul, République de Corée, lors de l'assemblée générale des membres de la Commission de l'OMT pour l'Afrique. Une résolution a été adoptée en appui à la réalisation de ce projet afin de faciliter l'obtention de financement auprès d'agences de développement international. À l'été 2003, un article faisant le point sur l'état d'avancement de l'étude était publié dans la revue TedQual de l'OMT. Un sommaire de cet article est présenté dans l'encadré ci-contre. 
Exemple de projet de recherche : le modèle de coopération dans la formation à distance pour l'enseignement du tourisme dans les pays en développement

L'utilisation des TIC dans la formation à distance a permis de faire reculer les frontières et de rejoindre des populations plus nombreuses, notamment celle des jeunes plus acquise à I'utilisation des technologies. Dans le cadre de la réduction de la fracture numérique entre le Nord et le Sud, la formation à distance devient un outil stratégique pour les pays en développement. Mais les initiatives internationales sont dispersées et gagneraient en efficacité si elles privilégiaient la coopération. Le mémorandum sur une entente de coopération entre I'Organisation mondiale du tourisme et le Groupe de la Banque mondiale, signé en 1999, a permis à une équipe de I'UQAM-CIFORT de proposer, en 2003, un modèle de coordination entre deux institutions de formation intervenant dans I'e-formation en tourisme dans les pays en développement, notamment en Afrique. Ces institutions de formation supérieure sont I'UQAM-IIFORT (Canada-Québec) et la George Washington University (É-U.).

Cette initiative en e-formation pour les pays africains s'appuie sur deux concepts développés par I'OMT, le GTAT' et le TedQual. . Ele vise sur le plan opérationnel, à numériser les contenus de cours GTAT pour les diffuser en ligne, ou par CD-Rom, et à étendre les programmes en tourisme des universités participantes. À ce jour I'UQAM et la George Washington University ont manifesté leur intérêt pour le projet.

Le projet vise à aider les universités d' Afrique participantes à acquérir la certification TedQual pour leurs programmes en tourisme, par l'amélioration des ressources humaines et le suivi de la formation assurée par I'UQAM et la George Washington University ou toute autre université participante membre du Conseil de l'éducation de I'OMT.
La figure ci-dessous donne un aperçu de l'opérationnalisation du projet. La numérisation des cours sera assurée par les institutions universitaires participantes au programme, ayant le label TedQual.

Le programme type en tourisme comprendra environ 24 cours en français et en anglais, adaptés et améliorés selon les réalités et les besoins de ces pays. Chaque partenaire africain sera jumelé à une université accréditée TedQual qui agira comme mentor. Cette approche est reconnue comme stratégique pour ce qui concerne la poursuite maximale des objectifs prévus par le programme. II s'agit ici de permettre au plus grand nombre d'universités africaines d'obtenir la certification TedQual pour leurs programmes en tourisme. D'autres institutions et organisations pouront contribuer à cette initiative, sous la coordination de l'Université du Québec à Montréal et de la George Washington University.

Les avantages liés à cette initiative sont les suivants:

- créer un réseau d'institutions de formation de haut niveau offrant des programmes accrédités TedQual et partageant, au moyen des TIC, leurs ressources et leur savoir avec des institutions de pays en développement pour leur permettre d'améliorer leurs programmes;

- favoriser le développement de partenaniats public-privé dans les pays en développement participants afin de faciliter la diffusion des cours GTAT à l'échelle locale.

Parmi les bénéficiaires du projet, citons les institutions de formation en tourisme, les autorités nationales du tourisme et les gestionnaires de l'industrie touristique.

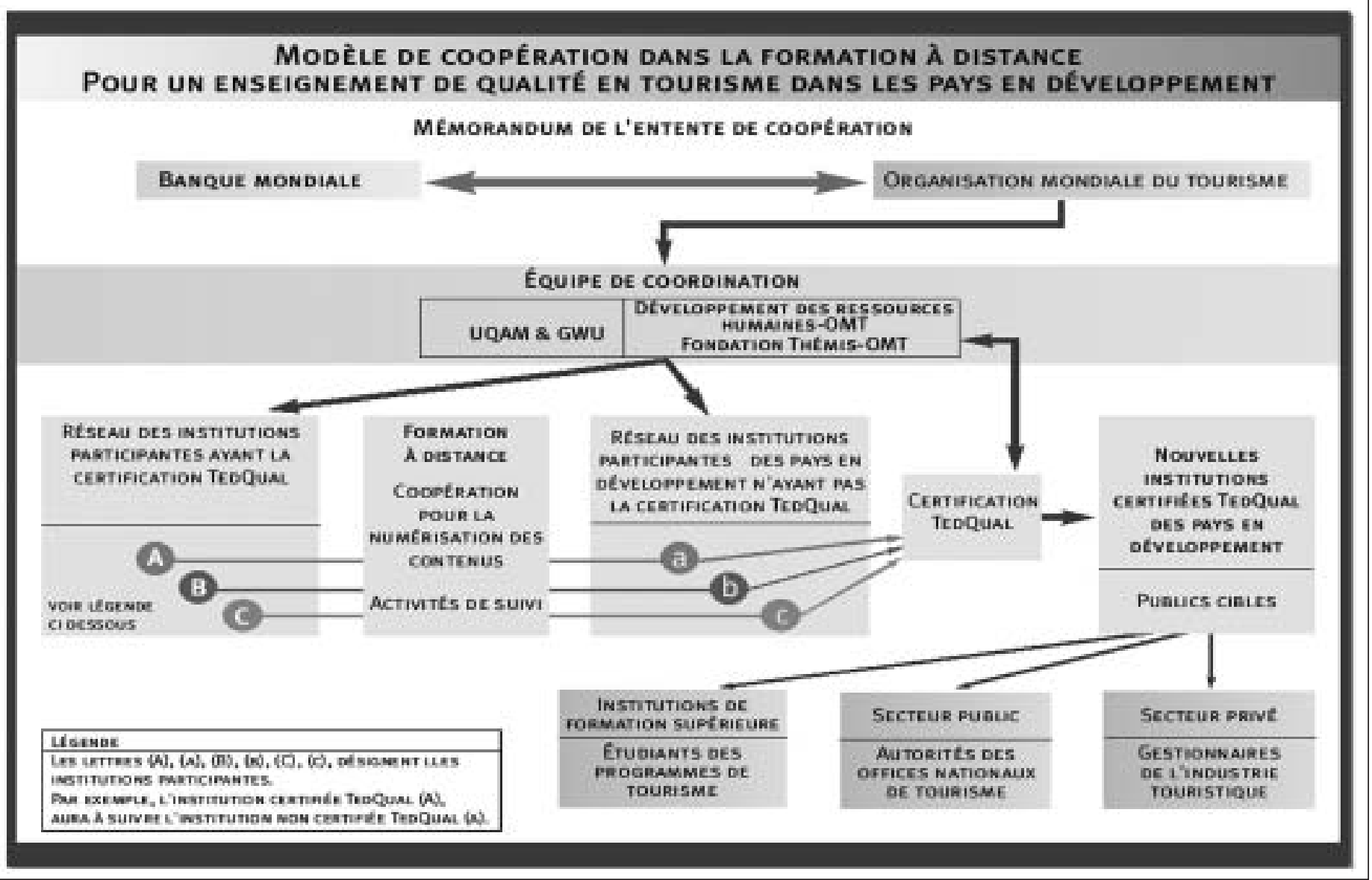

Source: François Bédard, UQ AM -C IFO RT (2003). 


\section{La veille en tourisme}

L'accès à l'information fiable et actualisée, dans un contexte de turbulences économiques et politiques et de changements technologiques, présente un caractère stratégique pour les intervenants de l'industrie touristique. L'implantation d'un réseau de veille en tourisme présente un intérêt certain pour les managers de cette industrie. Ainsi, la Chaire de tourisme de l'UQAM, engagée depuis 1992 dans la construction et le renforcement d'une relation étroite université-industrie touristique, a inauguré le réseau de veille en tourisme au début de 2004. Utilisant les nouvelles technologies pour rejoindre les entreprises touristiques, cette initiative vise particulièrement « à repérer, collecter, analyser et diffuser de l'information à valeur ajoutée afin d'accroitre la compétitivité de l'industrie et la vision prospective de ses dirigeants »(Chaire de tourisme $\left.{ }^{3}\right)$. Ce projet a reçu un appui financier de l'ordre de 1,5 million de dollars sur trois années, provenant de Tourisme Québec (650 000 \$) et de Développement économique Canada (950 000 \$).

\section{Le développement local durable et le tourisme}

Le tourisme est perçu par les grandes organisations comme un outil privilégié de la lutte contre la pauvreté et comme un élément important du développement local durable. Deux projets importants sont identifiés.

\section{Développement local durable et tourisme au Honduras}

L'état de pauvreté s'exprime d'une manière flagrante dans certains pays de l'Amérique centrale, notamment au Honduras. Cette situation impose une mobilisation des communautés locales. Les universités honduriennes sont concernées par la situation, mais leur contribution au développement des communautés locales défavorisées est lourdement handicapée par un manque de ressources, telles que : « [..] la formation d'intervenants locaux spécialisés, support à leur organisation, l'assistance-conseil à l'analyse et l'évaluation de situations, à l'élaboration de projets [...] à la préparation de plans de développement, à l'organisation du suivi de réalisation ». Actuellement, les institutions universitaires honduriennes répondent très partiellement à ces critères. Néanmoins, un renforcement institutionnel d'une université hondurienne, l'UNITEC (La Universidad Technologica Centroamericana), permettrait à cette dernière de jouer un rôle moteur dans le domaine du développement local. Le projet de jumelage entre l'UNITEC et l'Université du Québec à Montréal a été préparé en ce sens.

Ce projet vise donc l'accroissement de la capacité des universités honduriennes à appuyer le développement des communautés locales défavorisées, dans une perspective de développement durable.

Soutenu par l'ACDI (Agence canadienne pour le développement international), le projet, initié par le professeur Paul Bodson, a reçu un financement de 750000 \$ pour une durée de cinq années.

\section{Développement local durable et tourisme au M exique}

Il s'agit d'un projet de recherche-action à Cholula, engagé par la Chaire du Canada en patrimoine urbain, en collaboration avec
l'INRS-Urbanisation, culture et société, et l'Université Pueblas Las Americas. Le projet porte sur la mise en valeur du patrimoine culturel et religieux de Cholula au Mexique et la réalisation d'un plan de développement des infrastructures d'accueil.

\section{Conclusion}

Les thématiques de recherche privilégiées par le CIFORT et ses membres pour la période 2004-2006 ne couvrent certes que quelques aspects du phénomène du tourisme. Néanmoins, ces thématiques représentent les grandes problématiques de l'heure que sont l'accès à l'information et à la connaissance et leur diffusion par les technologies pour réduire l'écart entre le Nord et le Sud et contribuer ainsi à un développement local durable. Ces aspects font aujourd'hui partie de l'agenda des grandes organisations internationales et des institutions de formation supérieure confrontées aux problèmes de développement, de formation et de réduction de la fracture numérique.

Boualem Kadri achève la rédaction d'une thèse de doctorat (Ph.D.) en études urbaines sur la métropolisation des villes arabes méditerranéennes et est actuellement chargé de cours au Département d'études urbaines et touristiques de l'École des sciences de la gestion de l'Université du Québec à Montréal.

\section{Notes}

1 Le concept des cours GTAT (General Tourism Achievement Test) a été élaboré par l'OMT en collaboration avec une équipe de formateurs. Le contenu de chaque cours est d'abord divisé en grands thèmes, puis en domaines de connaissances, en thèmes et en sous-thèmes. Ces cours ont été conçus pour répondre aux besoins de formation du personnel des Administrations nationales du tourisme membres de l'OMT.

2 La Certification TedQual de l'Organisation mondiale du tourisme (OMT) est un système unique d'assurance de la qualité offert aux institutions, aux universités, aux écoles de gestion et aux centres de formation reconnus internationalement. Source : www.themis.ad (consulté le 15 mars 2004).

3 Présentation du réseau de veille organisée par la Chaire de tourisme, le 25 février 2004, à l'UQAM.

\section{Bibliographie}

Bédard, François et al. (2003), Centres d'initiatives facultaires sur l'intégration des technologies de l'information et de la communication, Rapport d'étape du projet FODAR-CIFITIC, Université du Québec à Montréal, septembre.

Bédard, François, Douglas C. Frechtling, et Donald E. Hawkins (2003), «E-Learning Cooperation Model for Quality Tourism Education in Developing Countries », TedQual Magazine, Organisation mondiale du tourisme, Madrid, Espagne.

Bédard, François, et Boualem Kadri (2003), Réduction de la fracture numérique en tourisme. Le rôle des grandes organisations internationales, Presses de l'Université du Québec, août.

Bédard, François, et Martine Géronimi (2002), L'ajustement aux technologies de l'information et de la communication : stratégies émergeant des attitudes et des comportements des enseignantes et des enseignants universitaires, Rapport d'étape sur l'enquête menée au printemps 2002 auprès du corps enseignant de l'UQAM [http://www.unites.uqam.ca/LABTIC/publications.htm]. Castells, Manuel (2002), La galaxie Internet, Paris, Fayard. 